\title{
Sperm Infertility Caused by Experimental Testicular Autoimmunity in the Nile Tilapia
}

\author{
Kazuhiko Mochida* and Hiroya Takahashi* \\ (Received July 29, 1992)
}

\begin{abstract}
Autoimmune responses against testis material were induced in mature male Nile tilapia Oreochromis niloticus, by injecting allogeneic testis homogenate emulsified with Freund's complete adjuvant. Some immunized males decreased their fertility 4 to 8 weeks after the first injection. Sperm motility of these fish decreased markedly. Ultrastructural observations of the testis and properties of spermatozoa in immunized fish suggested that this reduction was mainly caused by agglutination of spermatozoa by means of anti-sperm autoantibody penetrating the seminal lobules. Eight weeks after injection, globate structures, which seemed to originate from unabsorbed FCA component, occurred throughout the testicular tissue of some fish, possibly resulting in an obstruction of a normal flow of milt in the testis and a failure of milt discharge in immunized fish.
\end{abstract}

In fish culture, one of the most important subjects is to establish the technique of producing infertile fish in order to prevent the overpopulation effect on growth of object fish. In mammals, it has been shown that infertility can be induced efficiently by immunizing animals with sperm antigens. $^{1)}$ In the immune reaction, spermatocytes and spermatids were shed from the germinal epithelium of the testis into the lumen of seminiferous tubles, ${ }^{2}$ and spermatozoa were reduced in number due to phagocytosis by macrophages invading the tubule lumen. ${ }^{3,4}$ Finally, testicular weight and the diameter of seminiferous tubules were reduced, ${ }^{5,6)}$ and temporal infertility was caused in these animals. ${ }^{7)}$ One of the factors involved in the induction of infertility was thought to be agglutination and immobilization of spermatozoa by anti-sperm autoantibodies occurring in the blood and subsequently entering into seminiferous tubules. ${ }^{8, \theta}$ )

Some attempts have been made to obtain infertile fish by experimentally inducing testicular autoimmunity in Atlantic salmon Salmo salar, ${ }^{10,11)}$ rainbow trout Oncorhynchus mykiss, ${ }^{12,132}$ and Nile tilapia Oreochromis niloticus. ${ }^{14,15)}$ In these fishes, following immunization with testis material, macrophages enter into the lumen of seminal lobules of the testis to phagocytize spermatozoa, resulting in the formation of giantcell granulomas in the lobule lumen. In affected testes, however, spermatogenesis proceeds normally along the wall of the lobules, ${ }^{11-14)}$ indicating the difficulty of applying this method to successful induction of infertility in fishes. On the other hand, Lou and Takahashi ${ }^{102}$ reported that the blood-testis barrier was broken down after immunization with testis material, and that autoantibodies entered into the lumina of seminal lobules and efferent ducts to agglutinate spermatozoa. According to these observations, infertility may be caused by the agglutination of spermatozoa, so it is necessary to investigate the fertility of autoimmunized fish. The present study was conducted to check the changes of fertility, motility, and morphology of spermatozoa in the Nile tilapia autoimmunized with testis material, aiming at examining the possibility of preventing overpopulation in tilapia brood stock by rearing autoimmunized males.

\section{Material and Methods}

\section{Animal}

Mature tilapia Oreochromis niloticus of both sexes used in the present study were maintained in indoor concrete ponds in the campus of the Faculty of Fisheries, Hokkaido University, at $25-30^{\circ} \mathrm{C}$ under natural light conditions, and fed on a commercial diet for trout culture (Nippai Fish Food Co.) two to three times a day.

\section{Immunization}

The induction of autoimmune responses in the testis was carried out according to the method described by Lou and Takahashi.15) Mature

* Faculty of Fisheries, Hokkaido University, Minato, Hakodate, Hokkaido 041, Japan（持田和彦, 高橋 裕哉: 北海道大学水産学部). 


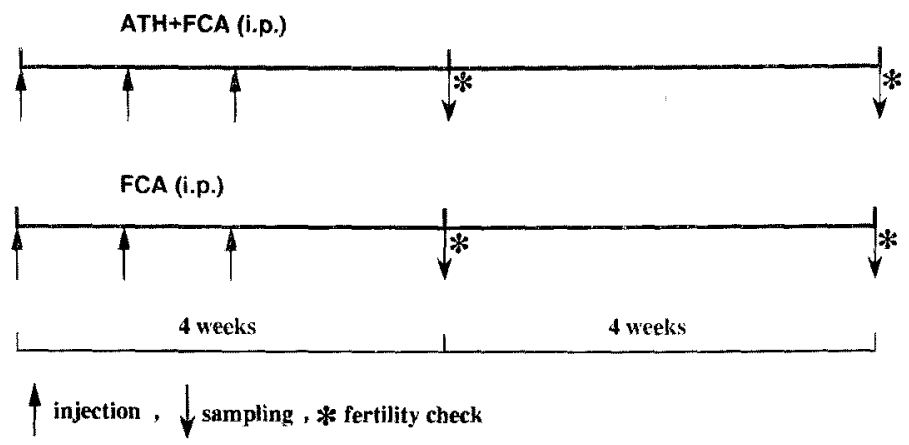

Fig. 1. Shedules of experiment.

male fish identified by their swollen genital papilla were used for experiments. Mature testis from freshly killed fish were homogenized at $4^{\circ} \mathrm{C}$ in a glass homogenizer with $0.7 \%$ saline to form a $50 \%$ solution of testis material. The allogeneic testis homogenate (ATH) thus obtained was emulsified in an equal volume of Freund's complete adjuvant (FCA). Eleven fish to be immunized received intraperitoneal (i.p.) injections of ATH + FCA, and the other 6 fish were injected with saline +FCA, at a dose of $2.5 \mu \mathrm{l} / \mathrm{g}$ body weight. Injections were given thrice at intervals of one week according to the schedule shown in Fig. 1. Eight intact male fish receiving no injection served as controls.

\section{Antibody Titration}

Sperm agglutination titer (SAT) was measured for sera obtained from both the immunized and FCA-injected fish to determine the intensity of autoimmune response in the testis, according to the method described by Lou and Takahashi ${ }^{13}$ ) with a slight modification.

All sera were decomplemented at $50^{\circ} \mathrm{C}$ for 30 min before use. Twenty $\mu l$ of the sera were serially diluted in phosphate-bufferd saline in microtiter plates. To each well of the plate was added an equal volume of fresh tilapia milt adjusted to $2.8-3.6 \times 10^{7}$ spermatozoa $/ \mathrm{ml}$ saline. After incubation at room temperature for $3 \mathrm{~h}$, the microtiter plates were kept at $4^{\circ} \mathrm{C}$ to settle spermatozoa. Controls for the SAT including both saline and the sera of intact tilapia were tested under the same conditions. SAT were determined by the formula, SAT $=-\log _{2} X$, where $X$ was the dilution of serum at which sperm agglutination became undetected.

\section{Assessment of Sperm Motility}

Spermatozoa in milt were obtained by gently pressing the abdomen of immunized male fish. Ten $\mu l$ of milt were diluted with $200 \mu l$ of water on a slide glass. Sperm motility was determined by measuring the duration until all spermatozoa had ceased to move in five different fields observed under a light microscope at a magnification of one hundred.

\section{Assessment of Sperm Fertility}

Measurement of sperm fertility was carried out 4 and 8 weeks after immunization. Immunized males were anesthetized with ethyl m-aminobenzoate methanesulfonate. Their milt was stripped out and sucked in capillary tubes (Terumo Plain). Milt of intact males served as a control.

Usually 10 mature females weighing about 200-300 $\mathrm{g}$ were kept in 60-liter glass aquaria with well-aerated and filtrated water regulated at $28 \pm 2{ }^{\circ} \mathrm{C}$. Ovulation was confirmed to occur in females which were building a nest and displayed a swollen genital papilla. A selected female was removed from the aquarium and anesthetized. After wiping the body thoroughly, the eggs of the female were stripped out into a small container. Milt in capillary tubes was diluted with about $5 \mathrm{ml}$ of water and stirred gently for a few sec. The sperm suspension and the stripped eggs were mixed and gently stirred with a writing brush for 10-20 sec. After settlement for $30-40 \mathrm{~min}$, the eggs were transferred to a closed-system incubator for cichlid eggs kept at $28-30^{\circ} \mathrm{C}$. Completion of fertilization was determined by the appearance of pigmentation on the egg surface 2 days after the artificial fertilization. Fertility of each milt was calculated by the formula, (number of eggs fertilized)/(total number of eggs tested: $X) \times 100$ 
$(\%)(X>100)$.

\section{Histology and Cytology}

Of the males used for the fertility test, some were killed and their testes were removed. Usually a median portion of each testis was cut into three fragments. One of the fragments was fixed in Bouin's fuid for light microscopic examination. Serial paraffin sections were cut at $5 \mu \mathrm{m}$ thick and stained with hematoxylin and eosin.

Another one was processed for electron microscopy. The testis fragment was cut into small pieces, fixed with a mixture of $1 \%$ glutaraldehyde$1 \%$ paraformaldehyde in $0.1 \mathrm{M}$ cacodylate buffer (pH 7.2) at $4^{\circ} \mathrm{C}$ for $12-24 \mathrm{~h}$, rinsed in the same buffer containing $10 \%$ sucrose for $3-6 \mathrm{~h}$ at $4^{\circ} \mathrm{C}$, then postfixed in $1 \% \mathrm{OsO}_{4}$ in the same buffer for $2 \mathrm{~h}$ at $4^{\circ} \mathrm{C}$. The specimens were dehydrated through a graded acetone series and embedded in Epon. Ultrathin sections (silver to grey) were cut with glass knives on a Reichert-Jung Ultracut E ultramicrotome, and viewed with a Hitachi H-7000 electron microscope after staining with uranyl acetate and lead citrate. Parallel $1 \mu \mathrm{m}$ sections of the specimens were also cut and stained with toluidine blue for the orientation of affected tissues.

The last one was embedded in OCT compound and frozen in dry ice-acetone. The sections were cut at $5 \mu \mathrm{m}$ thick with a cryostat (ReichertJung) and stained by immunofluorescence to detect the autologous IgM. After being blocked with $3 \%$ BSA, the specimens on a slide were incubated with rabbit anti-tilapia IgM antiserum for $1 \mathrm{~h}$ at room temperature, washed three times with PBS, and incubated for $1 \mathrm{~h}$ at room temperature in a dark humid chamber with FITCconjugated goat anti-rabbit IgG antibodies (Sigma, dilution 1:80). The slide was observed under an Olympus BH microscope equipped with epiffuorescence. Rabbit anti-tilapia IgM antiserum was obtained by the method described before. $^{172}$

In the present study, stages of spermatogenesis and spermiogenesis were determined according to the criteria proposed by Grier. ${ }^{182}$ Live spermatozoa obtained by stripping immunized and intact male fish were processed for scanning electron microscopy. Spermatozoa in milt were directly fixed by adding an equal volume of $2 \%$ glutaraldehyde to milt. Small pieces of $\mathrm{gel}$ bond film (Pharmacia) pre-coated with $0.1 \%$ poly-L-lysine were immersed in this sperm sus- pension. After $1 \mathrm{~h}$, the gel bond films were immersed in $1 \%$ glutaraldehyde for another hour, and dehydrated through a graded ethanol series. After ethanol had been replaced by tertbutyl alcohol, the specimens were freeze-dried, sputter-coated with gold $(25 \mathrm{~nm})$, and viewed on a Hitachi S-2300 scanning electron microscope.

\section{Result}

Sperm Fertility in Fish Immunized with $A T H+F C A$

Changes in sperm fertility, sperm motility, and SAT in each individual of both the control and immunized fish are shown in Fig. 2. Four weeks after immunization, SAT showed a high titer of 10-12 in immunized fish except fish No. 3, showing a successful induction of autoimmune response to testis material. Sperm fertility of these fish was lower than that of controls. The fertility of fish Nos. 4 and 6 was at especially low values of 1.6 and $5.1 \%$ respectively. White granular bodies, which seemed to be aggregated spermatozoa, were seen in the milt of these fish, and more than $95 \%$ of spermatozoa showed no motility. Sperm motility of fish Nos. 1, 2, and 5 was observed to be 1,2 , and $4 \mathrm{~min}$, respectively, being clearly lower than that of controls. Fertility and motility of spermatozoa of fish No. 3 were similar to the values obtained in controls. SAT of fish No. 3 was about 5 , being slightly lower than that of the other five fish.

Three of the five fish could not release their milt when stripped 8 weeks postimmunization. Sperm fertility of fish No. 1 was only $2.1 \%$. The fertility of fish No. 2 was $60 \%$, and was slightly lower than that of controls. SAT of fish Nos. 1 and 2 was 12 and 5 , and sperm motility of these fish was 3 and $12 \mathrm{~min}$, respectively.

Changes in sperm fertility, sperm motility, and SAT of control and FCA-treated fish are shown in Fig. 3. SAT of the fish treated with FCA alone was kept at about three, 4 to 8 weeks after the first injection. At 4 weeks, sperm fertility of fish Nos. 1 and 2 was $58.5 \%$ and $71.7 \%$, respectively, being similar to those of controls, whereas that of fish No. 3 was $24 \%$. Sperm motility of these fish was around $6 \mathrm{~min}$, and was not significantly different from that of controls moving for about $9 \mathrm{~min}$. Eight weeks after injection, two out of three fish could not discharge their milt when stripped. Fertility and motility of spermatozoa of the remaining fish were $87.3 \%$ and $6 \mathrm{~min}$, respectively. 

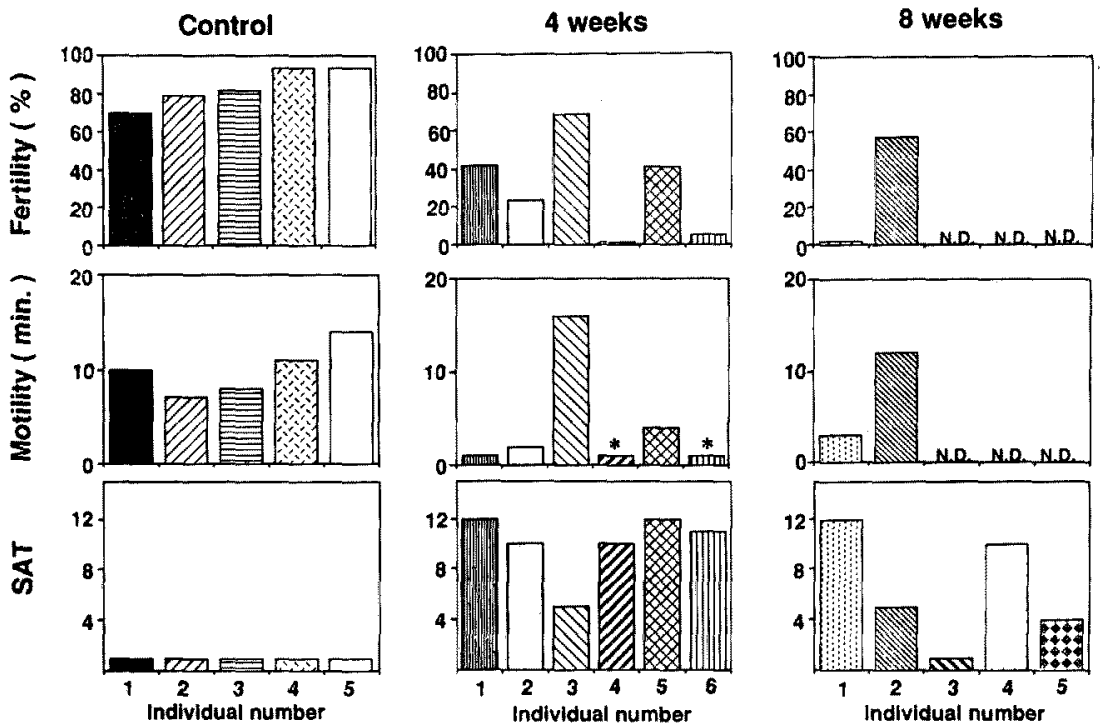

*, More than $95 \%$ of sperm did not move. N.D., milt could not be stripped.

Fig. 2. Fertility, motility, and agglutination titer (SAT) of sperm of each individual injected with ATH +FCA (i.p.) 4 and 8 weeks after the first injection.
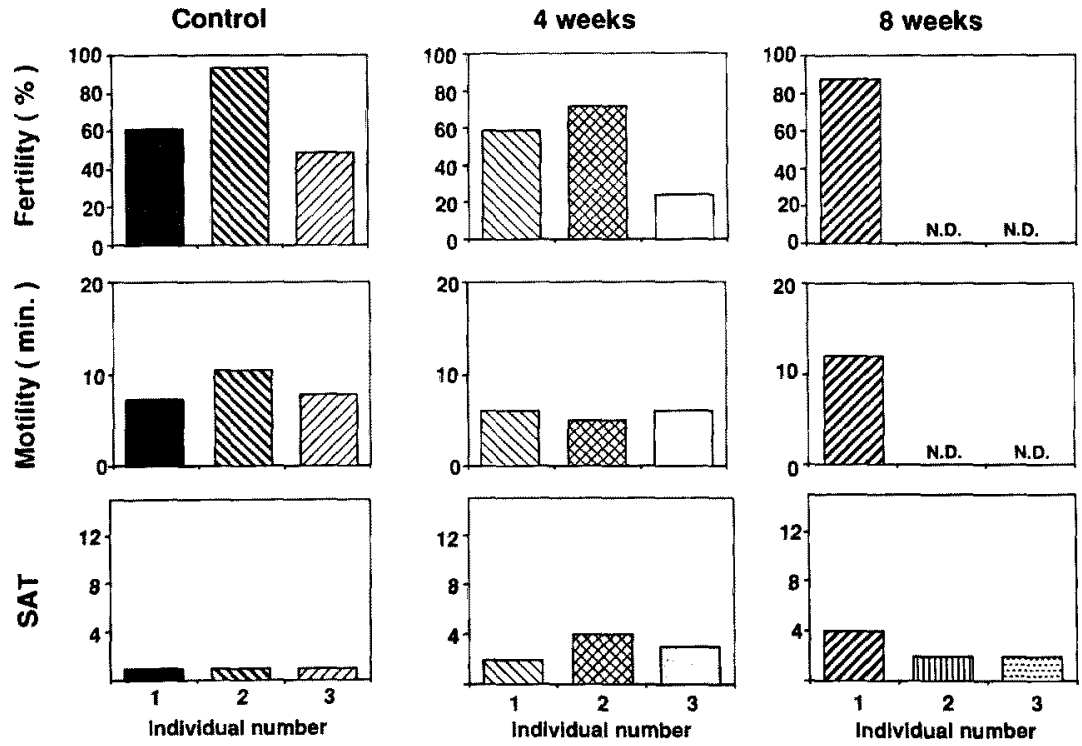

Fig. 3. Fertility, motility, and agglutination titer (SAT) of sperm of each individual injected with saline + FCA (i.p.) 4 and 8 weeks after the first injection.

\section{Autologous IgM Detection}

An attempt was first made to detect auto$\operatorname{logous} \operatorname{IgM}$ in the testis of intact tilapia by the use of immunofluorescence staining. Immu- nostaining was stronger in the testis capsule than in all other parts of the testis, and was slightly stronger in some interstitial areas than in the other part of the interstitium. Apparent fluorescence 
was detected in the cysts containing spermatogonia and spermatocytes, but not in those containing spermatids or in the lumina of seminal lobules and efferent ducts.

Four weeks after immunization, apparent fluorescent spots were observed especially on the wall of efferent ducts. In some of the lumina of seminal lobules and efferent ducts, at which free spermatozoa were present, weak fluorescence could be detected. Eight weeks after immunization, the fluorescence observed on the wall of efferent ducts became much stronger. In some parts, strong fluorescence was observed to extend along the wall of the duct. Spermatozoa in the lobule lumina were stained much stronger than those seen 4 weeks after injection with ATH + FCA (Fig. 4). The fluorescence was not detected in all but in most of the lumina of the lobules. In the testis of immunized fish, fluoresence in the cysts containing spermatogonia and spermatocytes was nearly the same in intensity as that found in the testis of control fish.

In groups of fish treated with FCA alone, the intensity of fluorescence in the testis was similar to that in control fish. In some of the fish, however, the wall of seminal lobules was strongly stained in some parts, and in these fish, weak fluorescence was detected in the lumina of the lobule and efferent ducts containing spermatozoa. In contrast to the case of the fish treated with ATH + FCA, fluorescence in the ducts of the fish injected with FCA occurred as spots or small masses. The testis of control fish, which were treated with normal rabbit serum or PBS instead of anti-tilapia IgM antiserum developed in rabbits, was not stained by the present immunostaining.

\section{Histopathological Changes in the Testis of Im- munized Males}

None of the immunized fish except for fish No. 1 showed any prominent pathological changes under a light microscope in their testes 4 weeks after immunization, though globate structures, which seemed to be an unabsorbed liquid paraffin component of FCA surrounded by thin layers of connective tissue, were observed to adhere to the surface of the testis. By electron microscopic observation, many branches of fibrils and also lymphocytes were shown to gather in this area. In the testis of fish No. 1, a number of eosinophilic cells appeared in the testicular tissue. They invaded the efferent ducts, and existed in clusters in the testis capsule. Globate structures were

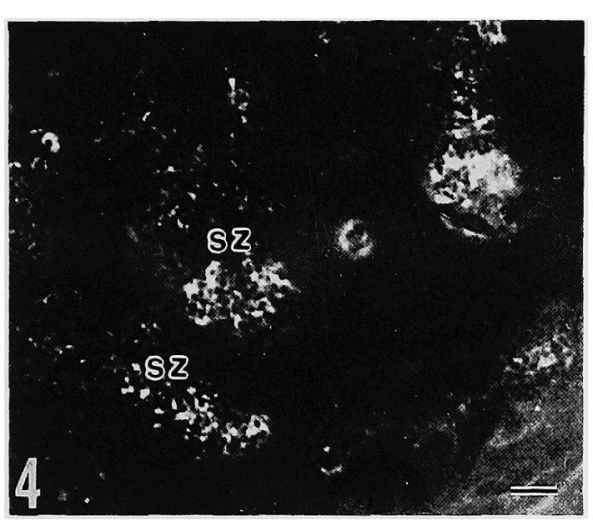

Fig. 4. Autologous IgM detected by immunofluorescence staining in the efferent duct of the testis of fish 8 weeks after immunization. sz, spermatozoa. Bar, $10 \mu \mathrm{m}$.

distributed all over the testis, and giant masses of cells, whose cytoplasmic matrices were not stainable with hematoxylin and eosin, surrounded the structures. Leucocytes slightly increased in number in the interstitial tissue of testis of immunized fish 4 weeks after immunization when compared with those in the testis of intact fish. Two types of leucocytes were prominent. One type was that of the lymphocyte, which was spherical in shape, about $6 \mu \mathrm{m}$ in diameter, and had a thin rim of cytoplasm containing prominent mitochondria, rough endoplasmic reticulum and some vacuoles. The other type was of the monocyte, which displayed an irregular contour. The cytoplasm contained some mitochondria, several endoplasmic reticulum, small vacuoles, and occasionally lysosomal bodies. The long elliptic-shaped granulocytes also existed in the interstitial tissue.

In most of the fish examined 8 weeks after immunization, lymphocytes were observed to invade seminal lobules and efferent ducts. Macrophages underwent phagocytosis of spermatozoa in the tubules. Leucocytes increased further in number in the interstitial tissue. Monocytes were more prominent in number than lymphocytes. In the interstitial tissue, monocytes maintained their spherical shapes, and measured 4-6 $\mu \mathrm{m}$ in diameter. They had well-developed Golgi apparatus, endoplasmic reticulum, lysosomal bodies, and sometimes several vacuoles. Some of these cells had no cytoplasmic processes. In their rather scanty cytoplasm, they were provided with mitochondria with clear matrix in the per- 

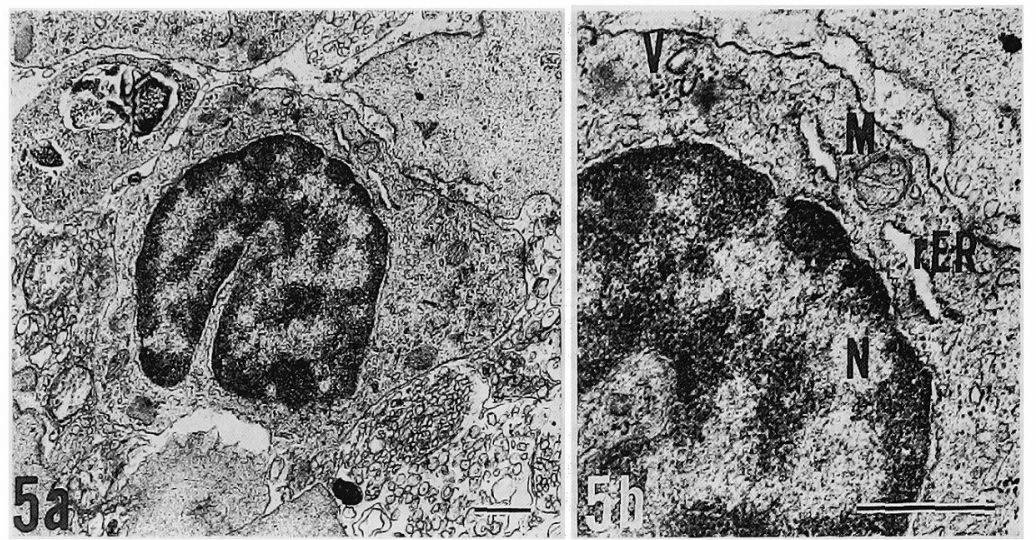

Fig. 5. Transmission electron micrograph of a monocyte (a) and its cytoplasmic organelles and inclusions (b) appearing in the interstitial tissues of testis from a male tilapia 8 weeks after immunization with ATH + FCA.

$\mathrm{N}$, nucleus; $\mathrm{M}$, mitochondrion; rER, rough endoplasmic reticulum; V, vacuole. Bars, $0.5 \mu \mathrm{m}$.

inuclear region and some small vacuoles along the cell periphery (Figs. $5 \mathrm{a}, \mathrm{b}$ ). Macrophages of a probable monocyte-line were shown to phagocytize spermatozoa. The phagocytosis of spermatozoa was observed only in the seminal lobules and efferent ducts. Globate structures surrounding FCA component came to invade the inside of the tesits. Many of them were observed to occur around efferent ducts.

In the fish injected with FCA, globate structures were observed in the testis, but pathological changes such as an invasion of lymphocytes into efferent ducts were not detected. In the fish 8 weeks after injection, these structures were widely distributed, especially around efferent ducts, and grew larger (Fig. 6). In both experimental groups, spermatogenesis proceeded normally even in the periphery of the globate structures.

\section{Agglutination of Spermatozoa in Milt of Im- munized Fish}

Spermatozoa stripped from immunized fish were observed with a scanning electron microscope. Large sperm agglutinations were frequently observed in the milt. The spermatozoa were mainly bound to each other by their heads (Fig. 7 a), though often the head was bound to the tail of other spermatozoa (Fig. $7 \mathrm{~b}$ ). Sperm agglutination of this type was regarded as a thangle type. The agglutinated spermatozoa were not different morphologically from normal spermatozoa obtained from control fish. In some parts of efferent ducts, there were some sper-

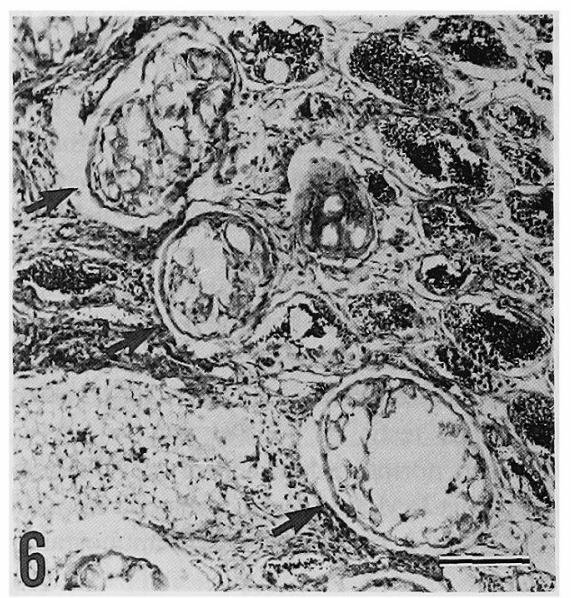

Fig. 6. Globate structures (arrows) observed on the testis capsule of fish injected with ATH +FCA. Bars, $50 \mu \mathrm{m}$.

matozoa which seemed to be agglutinated. They were bound mutually by some portions of their heads. Many other spermatozoa were found to be morphologically changed, and often expanded their cytoplasm containing some vacuoles. In fish treated with FCA alone, sperm agglutinations were observed neither in milt nor in the testis.

\section{Discussion}

The present results indicate that spermatozoa became functionally infertile in the Nile tilapia 


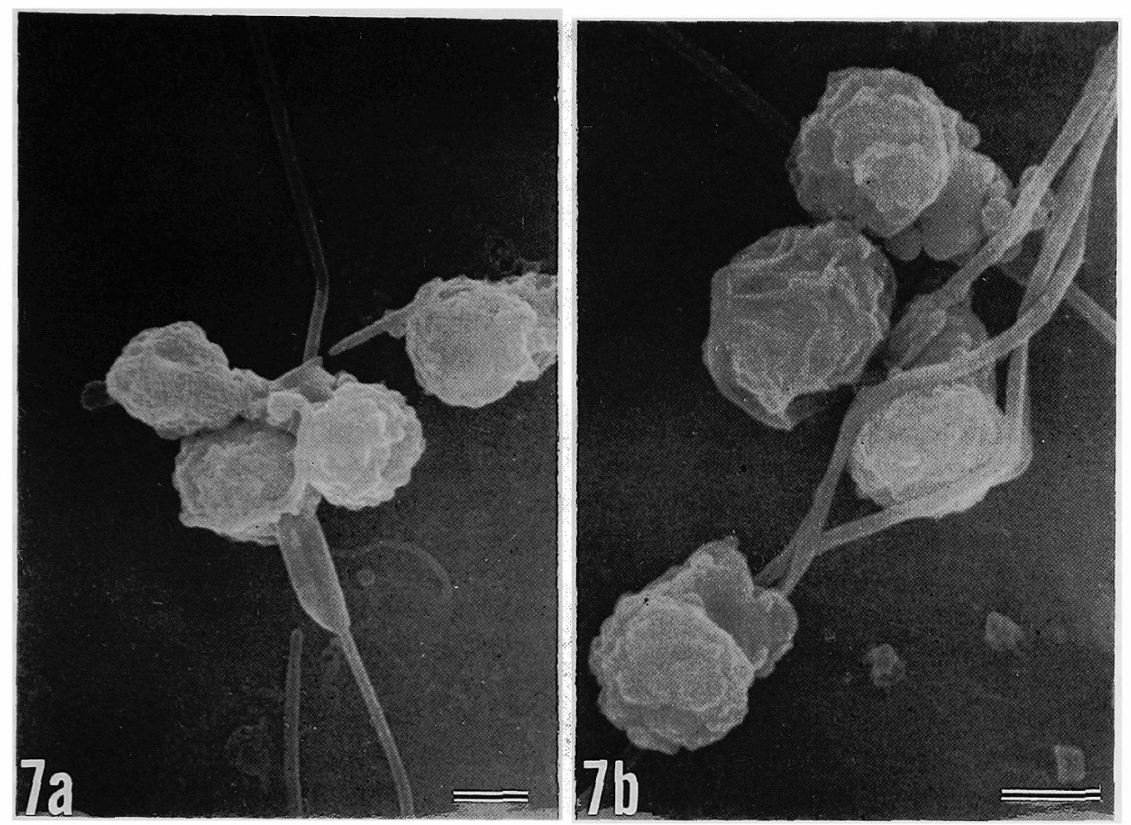

Fig. 7. Agglutination of sperm in milt stripped from fish immunized with ATH+FAC.

Spermatozoa were agglutinated in a head-to-head manner (a) or with a head-to-tail manner (b). Bars, $1 \mu \mathrm{m}$.

after treatment with testis material to induce testicular autoimmunization. Possible factors causing the infertility were a strong inhibition of sperm motility and a failure of milt discharge. These two factors may have a correlation with increased sperm agglutination titer which is an index of the intensity of humoral immunity.

In autoimmunized fish, the suppression of sperm motility seems to be caused mainly by the following reason. Agglutination of spermatozoa in milt was recognized as white granular masses when stripped from immunized fish. The occurrence of sperm agglutinations was confirmed by scanning electron microscopy of the milt. The spermatozoa were aggregated mainly in a head-to-head manner, which invariably makes them fail to fertilize the egg. It seems likely that the sperm agglutination results from an influence of autologous IgM which comes to penetrate the efferent duct as autoimmune responses in the testis progress. Autologous IgM was not immunohistochemically detectable in the efferent duct in control fish. In immunized fish, however, autologous IgM was shown to be attached to the head of spermatozoa. Lou and Takahashi ${ }^{10)}$ showed that, in the Nile tilapia, autoantigens existed locally on the head of spermatozoa as determined by the immu- nocytochemical method using anti-sperm autoantibody purified from the serum of immunized fish. From the results, it is suggested that autologous IgM penetrated into the efferent duct is the anti-sperm autoantibody which is generated in the blood by immunization against homologous testis material.

Naz and Mehta ${ }^{20)}$ described the ability of sperm antigens to induce cell-mediated immune response related to the testicular autoimmune response in the mouse; lymphocytes, which were obtained from animals immunized with sperm antigens and successively cultured in vitro, secreted several kinds of cytotoxin-like materials by the addition of a variety of sperm preparations to the medium. The materials activated macrophages and significantly inhibited sperm motility. In the present study, some spermatozoa in the efferent ducts and seminal lobules of immunized fish had expanded cytoplasm containing some vacuoles. This change seemed to be caused by disruption of the plasma membrane possibly by means of a penetration of some chemical components. Further, Sertoli cell phagocytosis of spermatozoa in immunized testes was significantly higher than that in normal testes (unpublished data). These results suggest that some mechanism that causes 
sperm infertility in the Nile tilapia may be in common with that in mammals in the case of induced autoimmune response against testis material, though there is no direct evidence for the secretion of cytotoxin by lymphocytes as obtained in the Nile tilapia.

A prominent reduction of sperm motility cannot completely explain the loss of sperm infertility in immunized Nile tilapia. In mammals, sperm surface autoantigens and isoantigens play an important role in successful fertilization at various stages from zona pellucida-sperm binding to embryonic development. ${ }^{21-23}$. Lou and Takahashi ${ }^{18}$ ) identified at least six kinds of sperm surface autoantigens in the Nile tilapia. At least one of them was suggested to be responsible for fertilization, since the fertility of spermatozoa coated with autoantibodies was apparently lower than that of spermatozoa coated with nonspecific antibodies purified from normal tilapia serum (unpublished data). Based on the assumption that sperm autoantigens are indispensably implicated in fertilization, it may be hypothesized that the autoantibodies penetrating efferent ducts and seminal lobules come to be attached to sperm surface autoantigens and block their function responsible for fertilization.

Eight weeks after immunization, 3 out of 5 immunized fish could not release their milt when stripped. Maturational stages of the testis of these fish were at IV or V, and their efferent ducts were filled with spermatozoa in some parts. In immunized rainbow trout, Secombes et al. ${ }^{123}$ demonstrated that granulomas fully filled the sperm duct and prevented normal spermiation 3 months after immunization. In the present study, granulomas were not formed in immunized testes, but many large and small globate structures were observed to occur throughout the testicular tissue 8 weeks after immunization. These globate structures might prevent a normal flow of sperm in the efferent ducts and seminal lobules.

Lou and Takahashi ${ }^{14)}$ showed that autoimmune response of the testis could not be induced to some degree by injections of FCA alone in the Nile tilapia. Although the precise mechanism of causing an immune reaction by FCA is obscure, the fact that the treatment with antigens emulsified with FCA can efficiently induce an immune reaction has been described repeatedly. ${ }^{24-203}$ In the guinea-pig, injection of FCA alone induced the breakdown of blood-testis barrier. ${ }^{27}$ In the Nile tilapia, if FCA actually affected the blood-testis barrier, autologous IgM would invade the efferent ducts and seminal lobules to directly or indirectly influence the sperm fertility of the fish. Four and eight weeks after the injection of FCA alone, autologous IgM was slightly detected in the efferent duct, and some junctional complex between Sertoli cells of the cyst wall became obscured. These results suggest that the blood-testis barrier was breached partly by the action of FCA itself. However, fertility and motility of spermatozoa of the treated fish was mostly unaffected. It is possible that testicular immune response cannot be induced efflciently by FCA alone at least in the Nile tilapia. The failure of milt discharge in two out of three FCA-treated fish could be explained by the fact that the testes of the fish had many globate structures originating from the component of FCA.

Many problems remain to be solved about the application of the induced sperm infertility for preventing the occurrence of overpopulation in tilapia culture. One of them is a variable intensity of testicular immune responses among immunized fish. In the present study, sperm motility and fertility in some fish were scarcely affected even after 8 weeks of immunization. Lou and Takahashi ${ }^{14)}$ pointed out that, at least in the Nile tilapia, the maturity of testes of the fish to be treated evidently has a critical importance in the induction of immune response to testis material. The maturity of testes of most of the fish used in the present study was at the stages from late IV to $V$ at the time of sacrifice, whereas that of the fish with indistinct immune reactions in testes was at the stages from III to early IV. At the time of immunization, the latter fish were immature, and there might be no spermatozoa bearing antigens on them in their testes.

Secombes et al. ${ }^{28}$ showed that monoclonal antibodies raised against spermatozoa of the common carp Cyprinus carpio and the rainbow trout could kill spermatozoa by complementmediated cytotoxity, and suggested a new method for the induction of infertility in male fish. They described some problems for the application of this method to aquaculture. First, when the guinea-pig serum as a source of complement is introduced into testis by intravascular injection, the serum will not affect the cell lysis if the antibody titer falls below a threshold dilution value. Second, as the complement-mediated cytotoxity is not expressed below $20^{\circ} \mathrm{C}$, the method cannot 
be applied to cold-water fish such as the rainbow trout. Their last argument may favor some warmwater fish such as the Nile tilapia.

\section{Acknowledgments}

The present authors wish to express their gratitude to Dr. Y.-H. Lou of Virginia University for his interesting discussion and to $\mathrm{Mr}$. K. Maekawa of the Donan Ezodai Shubyo Center for his technical advice on artificial fertilization of the Nile tilapia. This study was supported in part by a Grant-in-Aid for Scientific Research from the Ministry of Education, Science, and Culture of Japan to H. T. (No. 02454076).

\section{References}

1) P. Primakoff, W. Lathrop, L. Woolman, A. Cowan, and D. Myles: Fully effective contraception in male and female guinea pigs immunized with the sperm protein PH-20. Nature, 335, 543-546 (1988).

2) C. J. Flickinger, J. C. Heer, S. S. Howards, J. R. Sisak, J. M. Gleavy, T. J. Fusia, L. D. Vailes, and H. H. Handley: Early testicular changes after vasectomy and yasovasostomy in Lewis rat. Anat. Rec., 227, 37-46 (1990).

3) C. J. Flickinger, J. C. Heer, D. Caloras, J. R. Sisak, and S. S Howards: Inflammatory changes in the epididymis after vasectomy in the Lewis rat. Biol. Reprod., 43, 34-45 (1990).

4) M. H. Johnson: Changes in the blood-testis bartier of guinea pig in relation to histological damage following isoimmunization with testis, J. Reprod. Fert., 22, 119-127 (1970).

5) J. C. Heer, C. J. Flickinger, S. S. Howards, S. Yarbro, D. R Spell, D. Caloras, and T. N. Gallien: The relation between antisperm antibodies and testicular alterations after vasectomy and vasovasostomy in Lewis rats. Biol. Reprod., 37, 12971305 (1987).

6) W. B. Neaves: The effect of vasectomy on the testes of inbred Lewis rats. J. Reprod. Fert., 54, 405-411 (1978).

7) W. P. Kennedy: The production of spermatoxins. Quart. J. Exp. Physiol., 14, 279-283 (1924).

8) A. C. Menge, N. E. Medley, C. M. Mangione, and J. W. Dietrich: The incidence and influence of antisperm antibodies in infertile couples on sperm-cervical mucus interactions and subsequent fertility. Fertil. Steril., 38, 439-446 (1982).

9) R. A. Bronson, G. W. Cooper, and D. L. Rosenfeld: Correlation between regional specificity of antisperm antibodies to the spermatozoan surface and complement-mediated sperm immobilization. Am. J. Reprod. Immunol. 2, 222-224 (1982).

10) L. M. Laird, A. E. Ellis, A. R. Wilson, and F. G. T. Holliday: The development of the gonadal and immune systems in the Atlantic salmon (Salmo salar L.) and a consideration of the possibility of inducing autoimmune destruction of the testis. Ann. Biol. anim, Biochim. Biophys., 18, 1101-1106 (1978).

11) L. M. Laird, A. R. Wilson, and F. G. T. Holliday: Field trials of a method of induction of autoimmune gonad rejection in Atlantic salmon (Salmo salar L.). Reprod. Nutr. Develop.
20, 1781-1788 (1980).

12) C. J. Secombes, E. A. Needham, L. M. Laird, A. E. Lewis, and I. G. Priede: The 1ong-term effects of auto-immunologically induced granulomas on the testes of rainbow trout, Salmo gairdneri Richardson. J. Fish Biol, 26, 483-489 (1985).

13) C. J. Secombes, A. E. Lewis, E. A. Needham, L. M. Laird, and I. G. Priede: Appearance of autoantigens during gonad maturation in the rainbow trout (Salmo gairdneri). J. Exp. Zool., 233, 425-431 (1985)

14) Y.H. Lou and H. Takahashi: Induction of autoimmune responses to testes in a tilapia, Oreochromis niloticus. Bull. Fac. Fish. Hokkaido Univ, 38, 14-26 (1987).

15) Y.-H. Lou and H. Takahashi: Chronic changes of autoimmune responses to testis material in male Nile tilapia, Oreochromis niloticus. Bull. Fac. Fish. Hokkaido Univ., 39, 201-209 (1988).

16) Y.-H, Lou and H. Takahashi; The blood-testis barrier and its breakdown following immunization to testis material in the Nile tilapia, Oreochromis niloticus. Cell Tissue Res., $258,491-498$ (1989).

17) Y.-H. Lou, A. Hara, and H. Takahashi: Induction of autoantibodies against spermatozoa by injection of allogeneic sperm in the Nile tilapia, Oreochromis niloticus. Comp. Biochem. Physiol, 94B, 829-836 (1989).

18) H. J. Grier: Cellular organization of the testis and spermatogenesis in fishes. Amer. Zool., 21, 345-357 (1981).

19) Y.-H. Lou and H. Takahashi: Highly specialized sperm surface antigens in the Nile tilapia, Oreochromis niloticus, as revealed by conventional antisperm antibody and autoantibody. J. Exp. Zool., 258, 255-262 (1991).

20) R. K. Naz and K. Mehta: Cell-mediated immune responses to sperm antigens: effects on mouse sperm and embryos. Biol. Reprod., 41, 533-542 (1989).

21) P. Primakof, H. Hyatt, and D. G. Myles: A role for the migrating sperm surface antigen $\mathbf{P H} 20$ in guinea pig sperm binding to the egg-zona pellucida. J. Cell Biol., 101, 2239 2244 (1985).

22) C. B. Metz and J. Anika: Failure of conception in rabbits inseminated with nonagglutinating, univalent antibodytreated semen. Biol. Reprod., 2, 284-290 (1970).

23) R. K. Naz, N. J. Alexander, M. Isahakia, and M.S. Hamilton: Monoclonal antibody to a human germ cell membrane glycoprotein that inhibits fertilization. Science, 225, 342-344 (1984).

24) N. A. Jones, J. T. Willson, and S. Katsh: Passive induction of allergic aspermatogenesis in the guinea pig. Anat. Rec, 166,326 (1970).

25) Z. Pokorna: Induction of experimental autoimmune aspermatogenesis by immune serum fractions. Fol. Biol., 16, 320-329 (1970).

26) S. N. Upadhyay, P. Thillakoothan, A. Bamezai, S. Jayaraman, and G. P. Talwar: Role of adjuvants in inhibitory influence of immunization with porcine zona pellucida antigen (ZP-3) on ovarian folliculogenesis in bonnet monkeys: a morphological study. Biol. Reprod., 41, 665-673 (1989).

27) J. T. Willson, N. A. Jones, S. Katsh, and S. W. Smith: Penetration of the testicular-tubular barrier by horseradish peroxidase induced by adjuvant. Anat. Rec., 176, 85-100 (1973).

28) C. J. Secombes, A. Van Winkoop, গ. G. M. Van Den Boogaart, L. P. M. Timmermans, and 1. G. Priede: Immunological approaches to control maturation in fish. 1. Cytotoxic reactions against germ cells using monoclonal antibodies. Aquaculture, 52, 125-135 (1986). 